\title{
Uncertainty and Randomness: A Holonic Approach
}

\author{
Horacio Paggi \\ Facultad de Ingenieria \\ Universidad ORT Uruguay \\ Montevideo, Uruguay \\ horacio poggilagmail.com
}

\author{
Fernando Alonso Amo \\ Facullad de Informática \\ Universidad Politécnica de Madrid \\ Madrid, España \\ falonso'âti.upmes'
}

\begin{abstract}
Numerous researches on the representation and handling of the uncertainty, starting with the pioneering works about probability of Laplace, Bernoulli, etc. to reach a substantial increase during the twentieth century have been made, with applications in many fields (for example, psychology, decision theory, etc.), including the artificial intelligence one (in this case, related to the multiagent systems). On the other hand, the notions of holon and infermon developed by Koestler, Sulis, Alonso, Pazos et al. have shown to be a very useful way of to model evolving complex dynamic systems. In this work the relationship between holons, informons, randomness and uncertainty (as the opposed to certainty) in a holonic system that reacts to events is analyzed.
\end{abstract}

Keywords: holon, informon, uncertainty, vagueness, probability, fuzzy logic

\section{INTRODUCTION AND PRELIMINARY CONCEPTS}

Holons process information that can be certain or uncertain in different degrees, being the whole Universe composed by interactions between holons and informons. Specifically, when the holon is a person, Lindley [[1] Preface] writes: "You are uncertain, to varying degrees, about everything in the future, much of the past is hidden from you, and there is a lot of the about which you don't have full information. Uncertainty is everywhere and you cannot escape from it". Even though authors such as Dubois [2], Möller and Beer [3] and others distinguish between uncertainty and randomness, both of them can be seen as the holon incapacity of to give precise answers or to make sure decisions in many cases. In this work we will discuss some aspects of the lack of certainty (let us call it noncertainty in order to use a term without extra connotations) and its relation with the holons and the informons

This work is organized as follows: next, an outline on the basic concepts of holon, informon, event and uncertainty is given. In the section III we present many of the already done researches about the uncertainty; then, in the fourth section, the holonic-informonic frame is applied to the uncertainty, an example is given in the section $\mathrm{V}$. The conclusions and future work altogether the bibliography complete this work.

\section{BASIC CONCEPTS}

\section{A. Holon}

In 1926 Smuts introduced the concept of holism, pointing that [4] through the "wholes" are created the variety and complexity in the universe. Later, continuing with the study of the "wholes", the Austrian philosopher Arthur Koestler coined the term holon. In his book "The ghost in the machine" (1967) [5] comments that the hierarchies are something fundamental in the Nature: by having a hierarchic organization a system becomes more resistant, easier to maintain, to regulate and to repair than an unorganized one. He also notes that the first universal characteristic of the hierarchies is the relativity: "whole" and "part" in the absolute sense do not exist anywhere [5], and what really happens is that entities present two faces: one showing a self-contained whole and another showing a dependent part. All the entities are a "whole" and a "part" at the same time, and to name them the term he proposed was holon, from the Greek holos (whole) plus the suffix on, as in proton or nelitron, suggesting a particle or part. I the nature, the organic systems have vitality, structure and purpose; they are able to adapt themselves and to react to external stimuli, which are achieved thanks to their holonic structure. In those systems there is an ontogenic aspect, in which each holon has any excellence in process capacity and survives thanks to it. There is also a phylogenic aspect: the holonic system survives because the combination of the unique process capacities (competences or core skills) of the holons is more powerful and flexible that the one of the individual members separately [6]. A hierarchy in the Nature is an increasing order of wholeness (from particles to atoms to cells, etc). A hierarchy in the Nature should be called a holarchy [7]. One of the main characteristics of the holons is your multiple granularities that are manifested through the replication in auto-similar structures of fractal type with multiple resolution levels: the holarchy. 
Hock [8] called the holarchy "caord" from chaos and order: "any complex, auto-organized adaptive nonlineal community, be physical, be biologic or social". Holarchy is then "any complex chaotically ordered".

The concept of holon has also received another names given by different researchers; for instance, Simon calls them "sub assemblies" [9] and "intermediate forms"; Ingsber "stable structures" and Hatvany "entities" (cited by [10]).

Turnbull [11] citing Mathews [12] distinguishes between strong holons and weak holons. Strong holons are the holons that can exist autonomously while weak holons are such that they cannot exist without the rest of the members of the holarchy. In this work we won't make any distinction in them.

All these previous ideas gave rise to the holistic paradigm that has extended to many aspects of our lives, recognizing that much of the properties of a complex, evolving system cannot be reduced to the properties of its isolated components and that the system as a whole determines in a meaningful way how will behave the parts of the system. For example, in Software Engineering the paradigm is instantiated as the object oriented software and software agents, and in the philosophy, complete cosmologies such as the Wilber's $[7,13]$ and $[14]$ ones have arisen.

Wilber proposed a cosmology (in the Pythagorean sense of the "kosmos" as the union of all the domains of existence, from the matter up to the spirit, not only the physical world) in which holons play a fundamental role: reality is formed by wholes/parts, that is, the holons (Wilber's first principle) [7]. The second principle is: every holon has four forces driving it permanently: agency, communion, transcendence and dissolution. The capacities that allow the holon to be a part/to be a whole are called horizontal capacities, the vertical capacities are the ones related to the dissolution (such as when a cell dissolves into molecules after died) and the transcendence, when the holon becomes part of something more complex (for example, when a set of molecules becomes a cell). The constant conflict between the need of to be a part in order to subsist and the autonomy imposes a real stress [15] that implies the search of a balance through any of the behaviors of the holon. His third principle is related to the emergence: holons emerge. A holon cannot be reduced to its components, in a creative way. Every level of complexity would be accompanied by a certain degree of conscience [16] (for example, for a reptile, conscious is formed by hunger, physiological and sensorial feelings). The rest of the principles aren't related to the concept of holon.

Laureyssens gave a physical interpretation (a topological one) to the concept and considers holons as discrete regions of the space such as a region penetrates another generating the holons [14].
Finally, in many fields of the physics the term holon is used as synonym of chargon. In this case, an only electronic excitation can be decomposed into two independent excitations, one contributing the spin (the spinon) and another bringing the charge (the holon) [17$19]$.

We will adopt the meaning given by Koestler and Wilber.

The concept of holon has been extensively applied in many industry fields [20] [21-24]

\section{B. Informon}

The word informon is the fusion of the Latin "informo" and the Greek "on" (being, entity). Translated could be something like "informational being" [25].

Two stances appear about the idea of informon, one of them associated with the conscience.

Unrelated with the conscience are basically the definitions of Sulis and of Alonso et al. Sulis starts considering what information is: the etymology of informon shows that information and informons are related in a very deep way. But what is information? Information would be [26] the process by which an organization internally generates meaning from the expression of meaningful signs and signals of the others organisms 1 with which it interacts and must be understood as an "efficacious agency that is capable of affecting the characteristics and, ultimately, the behavior of some other. Information may influence the course of events but it does not necessarily cause them to occur" [26]. For Sulis, everything that informs exists ("existence principle") and everything that exists informs ("equivalence principle"). In an ontology based in these principles, the information urit (the most basic piece of information or reality) is the informon. More formally:"an informon is an aspect of reality that possesses the capacity to inform. An informon is an aspect of the reality that exist prior to any interpretation (semantic frame)" [26].

In the same line of ideas is the definition given by Alonso et al. [27]: "informon is the basic element of information that has sense for a holon and that allows it to make the right decisions and to execute the proper actions"

The correlation between this definition and the Sulis' one is clear: in both of them the information is a unit of (or that gives) meaningful information that affects the behavior of an entity.

\footnotetext{
1 Sulis gives an example showing that not only the living systems respond to the meaning of the signals that surround them, but also the physical (inanimate) ones.
} 
To end with this type of definitions, we want to mention a historically interesting definition of informon that was used by Utley in 1970: a kind of neural network, a modified Rosenblatt's perceptron that uses an unsupervised learning algorithm. This holon was used for adaptive pattem recognition [28-29].

Related to the conscience, Zeleznikar [30] poses that an informón is a conscious emergent informational entity. Informational entities (that is, that gives information) are characterized by three principles: every entity is informed by another one, informs to another and informs itself about itself. As an example, let us consider a certain word $\alpha$ from a thesaurus and the chain of words formed by the linked words that appears in the thesaurus (synonyms, antonyms, etc.) together the information contained for each of these words. The entity obtained considering the chain of linked informational entities (words) and the associated information would be the informón of $\alpha$ [25]. Zeleznikar also imposes that the informon is conscious, so we can consider this definition as a special case of the Sulis 'one; additionally, an informón will never be a simple piece of data for him, because a datum cannot be informed by an outside entity.

\section{Holons viewed as informons}

Besides the functional relationship existing between holons and informons in any information system, in which one Holon uses at least one informon (in the sense of Alonso et al.) to be able to work [27], there exists a relationship of dependent existence between the concepts of holon and informon, meaning this that there cannot exist a holon without informons (for example, corresponding to its status or its neighborhood's status) neither informons without holons (because of the definition of informon). Given that every holon can be considered as an informon, but not conversely, and given that Wilber assigns degrees of conscience to holons, many informons could have a certain level of conscious, as Zeleznikar states in his definition.

\section{More specifically:}

- By the Sulis definition, an informón is any aspect of the (not necessarily physical) reality. Given that a holon is a collaborative entity, by definition, it should communicate to the rest of the holons what are its central skills, at least. By making public this information, the holon is informing and, hence, is becoming an informon. On the other hand, many informons (those that correspond with autonomous entities that inform) can be seen, trivially, as holons (even though its answer to a collaboration requirement is "I do not collaborate").
- Considering the definition of Alonso et al.: instead of the holon is the information about the central skills of a holon which becomes an informon when used by another holon. Also, any other aspect of the holon can be an informon (for example, the description of the algorithm that the holons performs) is information for any other holon.

In the information systems, a holon as a set of bits will be an informon for another holon (such as the data storage manager) [27] and, additionally, a set of bits (an informon for some holon) could have certain process capacities and autonomy which are proper of a holon.

\section{Entity}

An entity is, in this context, a generator of informons [31]. Entities generate collections of informons that form dynamically coherent histories. Some of the generated informons (the public ones) could be made explicit through observation and even measurement. In this work we only consider this kind of informons. To assume the existence of the entities allow us to simplify the theoretical frame of holons and informons [31] so that the informons can be analyzed in dynamically coherent histories (another informons, as we will see). These entities impose a first level of meaning to the generated informons: the meaning is that the latter were generated by the former.

A semantic frame is a conceptual system, an organizational principle [31] that analyzes and groups phenomena in different entities, modes of being, modes of behavior, modes of acting and interacting and assigns meaning to all that in a coherent and consistent way. The semantic frame models and guides the interactions between the holons and those phenomena allowing keeping the thread of the meaning; it is a primitive construction such as the notion of set and more than to describe a particular process, it depicts a meaningful coherence of processes. Every holon interprets (assigns meaning to) the informons using different semantic frames.

\section{Event}

An event will be, as in the ordinary sense of the word, anything that happens, that occurs. Kolmogorov [32] uses the term in this sense. An event will exist as long it is meaningful for someone, that is, if it generates informons that influence the behavior of some holon ("Equivalence law": I inform, therefore, I exist [26]).

Events can be thought as decomposing/grouping themselves in/from other events; Kolmogorov refers to this when talks about "events" and "elemental events" [32] and so suggests De Finetti [33] with the idea that a 
"case" can be expressed as a combination of so many events as necessary. Some sets of holons will form another holon, which in turn can interpret some sets of events as other events. In the Section II the importance of this stratification of holons and informons will be clarified.

\section{E. Uncertainty}

For the Merriam-Webster Online Thesaurus [34], uncertainty is "a feeling or attitude that one does not know the truth, truthfulness, or trustworthiness of someone or something <uncertainty about her job prospects has her worried> - see Dol|BT". Hence, when referring to uncertainty, one is talking about a continuum that ranges from the absolute certainty to the impossibility. Uncertainty can be associated to a state of a subject with which it must make decisions, that must be represented and communicated [35]. In the literature appear terms such as variability, vagueness, ambiguity and imprecision as related to uncertainty. Variability is linked to randomness, chance and the analysis of the information coming from an expert, it would be studied using the probability theory methods [3]; vagueness would be typically an uncertainty referred to a linguistic expression, where the possible states about the inclusion are not clearly defined (e.g.: "to be young": we cannot define clearly the age until one is young). It doesn't have statistical properties and always have a part of subjective content [3]. Imprecision is usually related to quantitative, measurable data and can be used to measure the lack of knowledge of descriptive entities (for example, "good data quality") [36]. Other classifications of the non-certainty are possible: historically, Coombs, Dawes and Tversky [37] distinguish two types of uncertainty, one related with the world states (which in turn correspond to decision under incomplete knowledge) and another one linked to the own decision maker (this conducting to decision under unsure preferences). As we can see, there can be multiple sources of uncertainty: De Finetti mentions many (se Section II), Veseley and Ramson [cited by [35]] distinguish two kinds of uncertainty: physical uncertainty, generally owned to the stochastic variability, the measure or the sampling error and, on the other hand, the lack of knowledge.

Additionally, Budescu and Wallsten [cited by [35]] states the existence of "precise uncertainties" and "vague uncertainties", the former being the one which can be expressed as a point estimation or through a second order probability distribution on the probability values, while the latter would be formed by the rest of the uncertainties. Lukasiewickz and Straccia refers to "probabilistic uncertainty" and "fuzzy vagueness" [38]. Given that the information (and the uncertainty) have a conditioning character of the holons behavior, given an information (that is, one or more informons), different holons will be influenced in possibly different ways by it/them, each holon with different certainties, different opinions about the likelihood of the implied propositions; this degree of influence will in turn change through the time.

As we can see, the uncertainty appears connected with the decision making process: uncertainty becomes "a property of the fact themselves" [Van Winterfeldt and Edwards, cited by [35]],[1]. This uncertainty has been expressed and handled with probabilities (for the variability), fuzzy sets (for the vagueness), etc.

\section{RELATED WORK}

The amount of research done on what is not known with certainty is huge, including the works of mathematicians such as Kolmogorov, Bernoulli, etc.; however, just in the last decades has been clarified (for example, [39] [2]) the difference between the noncertainty due to the observer (ignorance: uncertainty) and the one "properly" owned to the observed phenomenon (what is classically called randomness). De Finetti considers "randomness" and "ignorance" as the same: "It makes no difference wether the uncertainty relates to an unforeseeable future, or to an unnoticed past, or to a past doubtfully reported or forgotten; it may even relate to something more or less knowable [...] but for which we are not willing or able to make the effort; and so on" [33]. Additionally, many works on the representation of uncertainty using specific knowledge representations have been done (see for example [40] referred to description logics used as knowledge representation).

Historically, the probability theory was the first form of giving a meaning to the randomness that a holon assigns to an event given many observed informons generated by the latter. From a philosophical standpoint probability can be understood as physical probability (frequentitst- Bernoulli, Von Mises, Pearson, etc.; propensity - Popper, Miller, Fetzer, etc; algorithmicSolomonoff, etc.) and evidential (epistemic- Bayes, Laplace, Cox, etc.; subjetivistic - De Finetti, Savage, etc.; Bayesian- Jayne, Bernardo, etc.) [41]. Good [cited in [36]] suggests that there are approximately 11 ways of interpreting probability, being the most remarkable: the classical theory, the frequentist theory and the subjetivistic one. Although the calculus is the same, the interpretation given to the probability influence the initial probabilities assignation (which is necessary to make operational the calculus) [36]. Complete analysis of these different interpretations can be found in [42], [36] and [43].

On the other hand, the uncertainty theories evolved from the fuzzy sets theory giving the fuzzy logic of Zadeh, intuitionist fuzzy sets of Kacprzyk, possibility 
theory and the generalized theory of uncertainty while others sought to generalize the epistemic probability theory (such as the imprecise probability theory of Walley [44], Cooman and Neumaier and DempsterShafer [45]). Recently, Vorobyev [46] proposed to unify the two theories (probability and uncertainty) in a framework that includes the holon observing the event. In this work, the "minds" (holons with a semantic frame in use) are the uncertainty providers while the events are governed by chance. Hence, two types of uncertainty exist for him: a) one generated by a set of "minds" (what is called generally uncertainty), measurable through the membership degree (different individuals can have different notions about the occurrence of an event), which comes from "the possibility of taking a probabilistic option among its own event sequence" [46] and b) another one generated by the events, that is measured with "probability" and that could be the random noncertainty. Minds and events can be grouped into sets, which mean to raise the ontologic/epistemic level in which one works, which in turn will produce an emergence of the no-certainties corresponding to that level. As can be seen, no certainty moves from one ontological/epistemic level to another although no endlessly, as it will be seen.

Given that random variables have fuzzy values, such a series of people watched in the street, who can be "young" or "old", the theory of fuzzy random variables appeared, started very initially by Goodman and later by Puri and Ralescu, Colubi and Gil.

In the Moller and Beer's work [3], the semantic frame, equated to an holon, is called a "model". Models could have the fractal self-similarity proper of the holons; uncertainty is owned to the model [3].

\section{THE HOLONIC-JNFORMONIC APPROACH}

Given the conceptual frame of holons-informons previously defined, an event will be an entity that generates different informons each of them having different meanings for the different affected holons. Its randomness will also depend on the observing holon (and, more specifically, on the semantic frame in use), and, in turn, given that holons are informs generators, ignorance can tum into randomness. This is the relation we can find between "randomness" and "ignorance" in a framework in which the judging individuals (the holons) are taken in account (which leads to a typically subjetivistic interpretation of the probability.

Randomness is not an intrinsic property of an event (or, equivalently, of an informón) but it emerges from the pair holon-informon, being the subjetivistic approach the most adequate here; probability would be "the degree of belief in the occurrence of an event attributed by a given person at a given instant with a given set of information" [33]. One must remember that the assignation of a degree of membership is also a subjective act. It is emergent in the sense that it cannot be reduced from the components (holon-informon) separately: it is a weak emergence [47].

Note that in the frequentist theories of probability, including Vorobyev's work, in order to compute the probabilities, the set of all possible events (informons) and "minds" (holons/agents) are supposed to be known. Given the dynamism in the holons formation and disintegration, and given that the set of informons managed by a given holon change through the time, the former supposition seems excessive.

A mechanism that allows building dynamically these relationships of non-certainty seems to be more adequate. The use of the Bayesian methods allows one approximation to it, incrementally through a two steps process: the holons and informons already known are considered, the no-certainties are calculated and then, typically when new informons appears (or when additional holons are taken in account), a review of the beliefs is made in order to update these no-certainties [48].

On the other hand, the holon-informon pair would form first order cybernetic systems. What is the role of the uncertainty in our framework? It can be considered as the decoupling between the semantic frame used to interpret the reality (environment) and the proper environment, which occurs naturally (without intervention of other holon) or because other holon influences the semantic frame (the "inertia law" of Sulis). Uncertainty, considered as ignorance or lack of knowledge [2] or weakness of a holon to decide or to act can require to be measured not only from a holon and the observed informon, but also a third component, a reference holon used to measure the ignorance of the original holon. Hence, uncertainty would be an emergent property of a second order cybernetic system. What is the result of considering the holon formed by the two holons observing the informon? A kind of randomness, coming from the holons uncertainties: the fuzzy randomness. Speaking in a more general way, what is obtained is a non-certainty which includes the randomness and uncertainties of each holon.

To consider randomness as an emergent property of a pair holon-informon and uncertainty as emerging from the pair holon-holon is another form of view the relationship between the theories of randomness and uncertainty (vagueness), because a holon can always be considered an informon. Molter and Beer refer as "the uncertainty phenomenon", which shows clearly this emergence. In the special case in which the holons are human individuals, "minds" in the Vorobyev nomenclature, we can think that the non-certainty ascends through the ontologic/epistemic levels. 
Observe that we are not stating how to measure the noncertainty, but we admit that is subjective. To measure it using a) probabilities (subjetivistic) with the respective use of the possibility logic [33] or b) uncertainty [1] assigning values ("the probability of $X$ ") to the degree of truth of the statement " $X$ is impossible" with the minimum $(0)$ for impossibility (false) and 1 for certainty (true)would be a confusion, in the opinion of Dubois and Prade [49] because the compositionality of the degrees of truth could not be applied to the degrees of belief.

\section{NON-CERTAINTY MEASUREMENT}

In this section we will not develop a theory of measure of non-certainty as it was already done with the probability theory, fuzzy sets theory, etc, but we are interested in how to measure it from the relationship between the holons using it of groups them in a dynamic form so the dynamic formation/dissolution of the holons is reflected in the used criteria. Turnbull [1011] proposed the Transactions Byte Analysis (TBA); the idea is that that minimizing the quantity of information (measured in bytes) transmitted between the holons one gets a structure that avoids the information overload and the "bounded rationality" problems. Intuitively, it seems that there could be a relationship between the quantity of exchanged data between holons and the beliefs they have in getting the utility. For example, if we think in terms of Shannon entropy (taking it as the degree of "surprise" that a message can produce) when coding the information with $\mathrm{N}$ bits, the maximum entropy is $\mathrm{N} / 2$, so, the more the bits used, more doubt/uncertainty in what can be received and, may be, a greater change in the belief of reaching the utility. The relationship between TBA and the decomposition seek here will be investigated in future works.

\section{AN EXAMPLE}

In [20] was depicted a holonic model of organization for the resolution of the incidents that takes place during the software projects development. The "solvers" of these incidents are holons formed by the help desk software (expert systems), people (technicians, groups of specialist, outsourced companies, etc.). These holonic "solvers" represent their knowledge by means of description logics rules, including axioms of the form $\mathrm{P}(\mathrm{A} \mid \mathrm{B})=x$ where $\mathrm{A}$ and $\mathrm{B}$ are two classes, meaning: "the probability of that an element a of A belongs to B is $x^{\prime \prime}$, as defined in [50]. For example, $\mathrm{P}$ (PCrunsSlow $\mid \mathrm{PChas}$ Virus $)=0.70$. These probabilistic rules are intended to express the uncertainties that the solvers have when dealing with a problem. Another classic approach would be to use fuzzy logic. As a third option, a whole set of plausibility functions, possibility functions, relative likelihood functions, etc. could be used [48]. In the three cases proper extensions of the description logics exist ( [5051], From an engineering point of view, it would be desirable the existence of reasoners supporting the chosen formalism used to represent the non-certainty (e.g. Pronto [52] for probability, FiRE [53], fuzzyDL [51] and FRESG [54] for a fuzzy approach and a prototype -GURDL- [54] for possibilistic logic have been created). Possibilistic reasoners implementing expressive description logics have been scarcely investigated yet, possibly because of the soundness and completeness they could pose [55]. Finally, to our knowledge, there are no reasoners implementing the fuzzy random variables theory.

All in all, by adopting the subjetivistic approach of the probability, all the probabilities can be assigned in the knowledge base rules of the form $P(A \mid B)=x$ with $A$ and $B$ classes, that is, the system can be immediately operative without the need of gathering hard data in order to compute the frequencies $x$. This option has also been taken in many engineering and science projects to minimize the testing times, to evaluate the performance of prototypes, etc. [36] so it's possible to make statements about the non-certainty of non-observable or unrepeatable events to incorporate information of all the sources deemed appropriated, to use moderate quantities of data obtained through the experimentation or observation and the incorporation of all the available knowledge in any given instant with the capacity of updating the probabilities as long there is more available knowledge. Basically, this subjetivistic approach is the interpretation of the non-certainty closest to the fuzzy logic [36]. Additionally, it can be shown [36] that the membership function can be used as the likelihood in the Bayes Theorem, which provides a mechanism of translation between the fuzzy logic and the probability theory. The other option for which ther are completely developed reasoners (fuzzy logic) would be perfectly acceptable, as it has been made in numerous cases (see for example [56]).

\section{CONCLUSIONS AND FUTURE WORK.}

In this work we have analyzed many aspects of the lack of certainty and the beliefs,

About the representation of the lack of certainty, we saw that the use of probabitities or fuzzy sets is not a dogmatic issue but it depends on the granularity level of the holons and informons.

This work is just an initial reflection about the indetermination and uncertainty using the holons and informons approach; many future works stems from it related to questions such as: 
a) How to calculate the belief (non-certainty) of the emergent holon, that is, from the noncertainties of the individual holons deduce the non-certainty that could have an emergent holon

b) How to perform a distributed reasoning using holons that represent uncertainty in different forms (for example, probability, fuzzy logic, plausibility functions, etc.).

\section{ACKNOWLEDGMENTS}

This work has been partially funded by the scholarship S-C-BE 55/18, Préstamo BID OCUR /1296PDT, Ministerio de Educación y Cultura, R.O del Uruguay.

\section{BIBLIOGRAPHY}

[1] Lindley, D., Understanding uncertainty. 2006: John Wiley \&Sons. pp. Preface, 12

[2] Dubois, D. "Uncertainty theories: A unified view". in SIPTA school 08 - UEE 08. 2008. Montpellier, France.

[3] Möller, B. and M. Beer, Fuzzy randomness. 2004: Springer.

[4] Smuts, J.C., Holism and evolution. 1926: Macmillan London \& NY. pp. 99-100

[5] Koestler, A., The ghost in the machine. 1967: Random House. pp. 40-50

[6] Mc.Hugh, P., G. Merli, and W. Wheeler, Beyond bussiness process reingeneering - towards the holonic enterprise. 1995, New York: John Wiley. pp. $80-82$

[7] Wilber, K., A brief history of everything. 2000; Shambala Publications Inc. pp. 27,39-40

[8] Hock, D.W. "Institutions in the age of minderafting". in Bionomics Annual Conference, 1994. San Francisco, California, USA.

[9] Simon, H.A., Models of a man, 1957, New York: John Wiley \& Sons.

[10] Turnbul], S., Grounding sociology in system science. 15th Armual Meeting of the Society du Travail (LEST). 2003, Aix en Provence, France.

[11] Turnbull, S., The governance of firms controlled by more than one board: Theory development and examples. Macquarie Graduate School of Maragement, Vol. Ph. D. 2000, Sydney: Macquarie University. 318.

[12] Mathews, J., Holonic organisational architectures. Human Systems Management. 1996. 27-54.
[13] Wilber, K., The integral vision: A very short introduction to the revolutionary integral approach to life, god, the universe, and everything. 2007: Shambala Publications Inc.

[14] Laureyssens, D. 2009 [cited 2009 19/11]; Available from: witw, mu6.com.

[15] Boardman, J. and B. Sauser, Systems thinking. Coping with 21st century problems. 2008: CRC Press.

[16] Wilber, K., The integral vision: A very short introduction to the revolutionary integral approach to life, god, the universe, and everything. 2007: Snambala Publications Inc. $p p$.

[17] Bernevig, B.A., D. Giuliano, and R.B. Laughlin, "Spinon-holon attraction in the supersymmetric $t-j$ model with $1 /$ r. Physical Review Letters", 2001. $87(17)$.

[18] Auslaender, O.M., et al., Spin-charge separation and localization in one dimension. Science. Vol, 308. 2005. 88-92.

[19] Koitzsch, A., et ai., "Current spinon-holon desctiption of the one-dimensional charge-transfer insulator srcuo2: Angle-resolved photoemission measurements. Physics Review", 2006. B 73.

[20] Paggi Straneo, H. and F. Alonso Amo. "A holonic model of system for the resolution of incidents in the software engineering projects". in 2009 International Conference on Computer and Automation Engineering. 2009. Bangkok, Thailand: IEEE.

[21] Chou, L.-D., K.-C. Shen, K.-C. Tang, and C.-C. Kao, Implementation of mobile-agent-based network management systems for national broadband experimental networks in taiwan, in Holonic and multi-agent systems for manufacturing. 2003, Springer Berlin / Heidelberg. pp. 280-289.

[22] Durna, M., A.M. Erkmen, and I. Erkmen. "The selfreconfiguration of a holonic hand: The holonic regrasp". in JEEE international Conference on Intelligent Robots and Systems. 2000.

[23] Liu, S., W.A. Gruver, and D.B. Kotak, "Holonic coordination and control of an automated guided vehicle system. Integrated Computer-Aided Engineering", 2002. 9(3): pp. 235-250.

[24] Ulieru, M. "Qualyntel: A holonic diagnosis and quality control system". in INFORM '98. 2004. Seattle, Washington.

[25] Zeleznikar, A., "Informon- an emergent conscious component. Informatica", 2002(26).

[26] Sulis, W., J. Nation, I. Trofimova, J. Rand, and W. Sulis, Archetypal dynamics, in Formal descriptions of developing systems. 2003, Kluwer Academic Publishers. pp. 180-227.

[27] Alonso, F., et al. "Fundamental elements of a software design and construction theory informons and holons". in International Symposium of Santa Caterina on Challenges in the Internet and Interdisciplinary Research. 2004. Amalfi, Italy.

[28] Uttley, A.M., "The informon: A network for adaptive pattern recognition. Journal of Theoretical Biology", 1970. 27: pp. 31-67. 
[29] Uttley, A.M., Simulation studies of learning in an informon network. Brain Res. Vol. 102. 1976. 3753.

[30] Zeleznikar, A. The philosophy and formalization of the informational. [cited 2009 19/1i]; Available from: has:/www artilico org/index.huml

[31] Sulis, W., Archetypal dynamics, in Formal descriptions of developing systems., J. Nation, et al., Editors. 2003, Kluwer Academic Publishers. pp. 180-227.

[32] Kolmogorov, A.N., Foundations of the theory of probability. 2nd. ed. 1956, New York Chelsea Publishing Co. pp. 2-5

[33] De Finetti, B., Theory of probability, I990: John Wiley \& Sons. pp. Preface, 4, 25-26, 30-3i

[34] Merriam-Webster. Merriam-webster online thesaurus. 2009 [cited 2009 19/11]; Available from: www merriam-webster.com.

[35] Gambara D'errico, H., "Incertidumbre y probabilidad subjetiva en la teoría de la decisión conductual. Revista de psicologia general y aplicada: Revista de la Federación Espantola de Asociaciones de Psicología", 1991. 44(2): pp. 199 208.

[36] Ross, T., J. Booker, and W.J. Parkinson, Fuzzy logic and probability applications: Bridging the gap. 2002: SIAM-ASA. pp. $57,63-69,88-89,97-98$

[37] Coombs, C.H., R.M. Dawes, and A. Tversky, Mathematical psichology: An elementary introduction. . 1970: Prentice Hall.

[38] Lukasiewicz, T. and U. Straccia, "Desctiption logic prograns under probabilistic uncertainty and fuzzy vagueness. International Journal of Approximate Reasoning", 2009. 50(6): pp. 837-853.

[39] Bobillo, F., M. Delgado, and J. Gómez-Romero. "Extending fuzzy description logics with a possibilistic layer". in Third ISWC Workshop on Uncertainty Reasoning for the Semantic Web. 2007. Busan, Korea.

[40] Bobillo, F., Managing vagueness in ontologies. Departamento de Ciencias de la Computación e Inteligencia Artificial. Vol. Ph. D. 2008, Granada: Universidad de Granada. 299.

[41] Vorobyev, O.Y., Eventology versus contemporary theories of uncertainty. XII International EM'2009. 2009. 13-31.

[42] Mellor, D.H., Probability: A philosophical introduction. 2005, New York: Routledge.

[43] Gillies, D., Philosophical theories of probability. Philosophical Issues in Science, ed. W.H. NewtonSmith. 2000: Routledge.

[44] Walley, P., Statistical reasoning with imprecise probabilities. 1991: Chapman and HaIl.

[45] Das, S., Foundations of decision-making agents. 2008: World Scientific Publishing Co. Pte. Tld.

[46] Vorobyev, O.Y. "Eventology of random-fuzzy events.". in IV All-Russian FAM'2005. 2005. Krasnoyarsk: Sib. Fed. Univ. .

[47] Abbott, R. "Emergence, entities, entropy, and binding forces", in The Agent 2004 Conference on:
Social Dynamics: Interaction, Reflexivity and Emergence. 2004.

[48] Halpern, J.Y,, Reasoning about uncertainty. 2003, Massachussetts, USA: MIT Press.

[49] Dubois, D. and H. Prade, Personal Communication,

[50] Baader, F., D. Calvanese, D. McGuinness, D. Nardi, and P.F. Patel-Schneider, The description logics handbook. 2007: Cambridge University Press.

[51] Bobillo, F. and U. Straccia, Fuzzydl: An expressive fuzzy description logics reasoner, 17th IEEE International Conference on Fuzzy Systems, ed. IEEE. 2008, Hong Kong, China: IEEE Computer Society. 923-930.

[52] Klinov, P., Pronto: A non-monotonic probabilistic description logic reasoner, in The semantic web: Research and applications, S.B. Heidelberg, Editor. 2008, Springer Berlin / Heidelberg. pp. 822-826.

[53] Simou, N. and S. Kollias. "Fire: A fuzzy reasoning engine for imprecise knowledge". in Ist. K-Space Ph. D. Students Workshop. 2007. Berlin, Germany.

[54] Hailong Wang, Z.M. Ma, and I. Yin. "Fresg: A kind of fuzzy description logic reasoner ". in 20th International Conference, DEXA 2009. 2009 Linz, Austria: Springer Berlin / Heidelberg.

[55] Simou, N.,H. Paggi,Personal Communication,1st sept 2009

[56] Ross, T.J., Fuzzy logic with engineering applications. 2nd. ed. 2004: John Wiley \& Sons. 